\title{
EFFICIENT MULTIPLE-TERM APPROXIMATIONS FOR THE GENERALISED ELLIPTIC-TYPE INTEGRALS
}

\author{
M. EL-GABALI ${ }^{1}$
}

(Received 12 December 1994; revised 27 February 1995)

\begin{abstract}
The generalised elliptic-type integral $R_{\mu}(k, \alpha, \gamma)$

$$
R_{\mu}(k, \alpha, \gamma)=\int_{0}^{\pi} \frac{\cos ^{2 \alpha-1}(\theta / 2) \sin ^{2 \gamma-2 \alpha-1}(\theta / 2)}{\left(1-k^{2} \cos \theta\right)^{\mu+1 / 2}} d \theta,
$$

where $0 \leq k<1, \operatorname{Re}(\gamma)>\operatorname{Re}(\alpha)>0, \operatorname{Re}(\mu)>-0.5$, is represented in terms of the Gauss hypergeometric function by Kalla, Conde and Hubbell [8]. In 1987, Kalla, Lubner and Hubbell derived a simple-structured single-term approximation for this function in the neighbourhood of $k^{2}=1$ in some range of the parameters $\alpha, \gamma$ and $\mu$. Another formula which complements the parameter range was recently derived by the author. In this paper a novel technique is used in deriving multiple-term efficient approximations in the neighbourhood of $k^{2}=1$ for $R_{\mu}(k, \alpha, \gamma)$ which may be considered as a generalisation to the concept of the single-term approximations mentioned above. Two non-overlapping expressions which almost cover the entire range of parameters $(\alpha, \gamma, \mu)$ are derived. Closed-form solutions are obtained for single- and double-term approximations (in the neighbourhood of $k^{2}=1$ ). Results show that the proposed technique is superior to existing approximations for the same number of terms. Our formulation has potential application for a wide class of special functions.
\end{abstract}

\section{Introduction}

Kalla, Conde and Hubbell [8] have treated a family of integrals of the form

$$
R_{\mu}(k, \alpha, \gamma)=\int_{0}^{\pi} \frac{\cos ^{2 \alpha-1}(\theta / 2) \sin ^{2 \gamma-2 \alpha-1}(\theta / 2)}{\left(1-k^{2} \cos \theta\right)^{\mu+1 / 2}} d \theta .
$$

These integrals have special importance since many physical and engineering problems are simply special cases of this representation, examples of which occur in radiation field problems $[2,5]$. Other forms of interesting elliptic-type integrals

\footnotetext{
'Kuwait University, Department of Mathematics, P. O. Box 5969 Safat 13060 Kuwait.
}

(C) Australian Mathematical Society, 1997, Serial-fee code 0334-2700/96 
are special cases of such representations. If, for example, $\gamma=2 \alpha=1, \mu=j$ (non-negative integer), then

$$
R_{j}\left(k, \frac{1}{2}, 1\right)=\int_{0}^{\pi}\left(1-k^{2} \cos \theta\right)^{-j-1 / 2} d \theta=\Omega_{j}(k)
$$

which is the well-known Epstein-Hubbell function [4]. First and second complete elliptic integrals are related to $\Omega_{0}(k)$, and $\Omega_{1}(k)$ respectively [8]. Kalla and Al-Saqabi [7] have treated the family of integrals

$$
K_{\mu}(k, n)=\int_{0}^{\pi} \frac{\cos ^{2 n} \theta}{\left(1-k^{2} \cos \theta\right)^{\mu+1 / 2}} d \theta
$$

which is related to the generalised elliptic-type integral by [1]

$$
K_{\mu}(k, n)=\sum_{m=0}^{2 n}(-1)^{m} 2^{m}\left(\begin{array}{c}
2 n \\
m
\end{array}\right) R_{\mu}\left(k, \frac{1}{2}, m+1\right) .
$$

If, $\gamma=2 \alpha$ then (1) gives

$$
R_{\mu}(k, \alpha, 2 \alpha)=2^{1-2 \alpha} S_{\mu}\left(k, \alpha-\frac{1}{2}\right),
$$

where

$$
S_{\mu}(k, v)=\int_{0}^{\pi} \frac{\sin ^{2 \nu} \theta}{\left(1-k^{2} \cos \theta\right)^{\mu+1 / 2}} d \theta .
$$

The case $\operatorname{Re}(\nu)=\operatorname{Re}\left(\alpha-\frac{1}{2}\right)>-\frac{1}{2}$ was studied by Kalla [6]. Al-Saqabi [1] has treated the family

$$
B_{\mu}(k, n, \beta)=\int_{0}^{\pi} \frac{\cos ^{2 n} \theta \sin ^{2 \beta} \theta}{\left(1-k^{2} \cos \theta\right)^{\mu+1 / 2}} d \theta,
$$

where $0 \leq k<1, \operatorname{Re}(\beta)>-\frac{1}{2}, \operatorname{Re}(\mu)>-\frac{1}{2}$, and $n$ is a non-negative integer, which is related to $R_{\mu}(k, \alpha, \gamma)$ by

$$
B_{\mu}(k, n, \beta)=2^{2 \beta} \sum_{m=0}^{2 n}(-1)^{m} 2^{m}\left(\begin{array}{c}
2 n \\
m
\end{array}\right) R_{\mu}\left(k, \beta+\frac{1}{2}, m+2 \beta+1\right) .
$$

In $[1,4-8]$ recurrence relations, series expansions, relations with known functions and asymptotic approximations in the neighbourhood of $k^{2}=1$ have been given. A simple-structured single-term asymptotic approximation in the parameter subspace where $\operatorname{Re}\left(\mu+\frac{1}{2}+\alpha-\gamma\right) \geq 1$ has been given by Kalla, Leubner and Hubbell in [9].

Another single-term approximation which covers the parameter subspace where $\operatorname{Re}\left(\gamma-\alpha-\mu-\frac{1}{2}\right) \geq 1$ has been derived by the author in recent work [3]. The results 
of [9] are included in [3] and the latter contains single-term asymptotic approximations of (1) which almost cover the entire range of parameters $(\alpha, \gamma, \mu)$. Our object in this paper is a generalisation of the work given in [3]. That is, we derive simply structured, efficient multiple-term approximations of (1) in the neighbourhood of $k^{2}=1$. It is sufficiently accurate to allow a semi-quantitative assessment of the dependence of (1) on the parameters $\mu, k \rightarrow 1, \alpha$ and $\gamma$, which is usually an important first step in physical applications [9]. The paper is organised as follows. In Section 2 two formulas for asymptotic expansions which almost cover the entire range of the parameters are obtained. Two general formulations of the multiple-term approximation are obtained in Section 3, while two expressions for single- and double-term approximations are given in Section 4, where different closed-form results are obtained. Finally, results, error estimates and concluding remarks are given in Section 5 .

\section{Asymptotic expansion}

2.1. Expansion valid for the parameter subspace $\operatorname{Re}\left(\mu+\frac{1}{2}+\alpha-\gamma\right)>\mathbf{N}, \mathbf{N} \geq \mathbf{1}$. With some simple transformations, (1) can be written as [9]

$$
R_{\mu}(k, \alpha, \gamma)=\frac{1}{\left(1-k^{2}\right)^{\mu+1 / 2} \kappa^{\nu-\alpha}} \int_{0}^{\kappa} \frac{x^{\gamma-\alpha-1}\left(1-\frac{x}{\kappa}\right)^{\alpha-1}}{(1+x)^{\mu+1 / 2}} d x
$$

For $\kappa=2 k^{2} /\left(1-k^{2}\right)$, the series expansion has been given in [9, Equation (6), p.273] by

$$
\begin{aligned}
& R_{\mu}(k, \alpha, \gamma) \\
& \approx \frac{\Gamma(\alpha)}{\left(1-k^{2}\right)^{\mu+1 / 2} \kappa^{\gamma-\alpha} \Gamma(\mu+1 / 2)} \sum_{n=0}^{N} \frac{(-1)^{n} \Gamma(\gamma-\alpha+n) \Gamma\left(\mu+\frac{1}{2}+\alpha-\gamma-n\right)}{n ! \Gamma(\alpha-n) \kappa^{n}}+R_{N},
\end{aligned}
$$

where $R_{N}$ is the remainder.

2.2. Expansion valid for the parameter subspace $\operatorname{Re}\left(\gamma-\alpha-\mu-\frac{1}{2}\right)>\mathbf{N}, \mathbf{N} \geq \mathbf{1}$. Equation (1) can also be written as

$$
R_{\mu}(k, \alpha, \gamma)=\frac{1}{\left(1+k^{2}\right)^{\mu+1 / 2}} \int_{0}^{\infty} \frac{\tau^{\alpha-1}(1+\delta \tau)^{-\mu-1 / 2}}{(1+\tau)^{\gamma-\mu-1 / 2}} d \tau
$$

(see [3]), where $\delta=\left(1-k^{2}\right) /\left(1+k^{2}\right)$. Its series expansion has been given in [3] as

$$
\begin{aligned}
& R_{\mu}(k, \alpha, \gamma) \\
& \quad=\frac{1}{\left(1+k^{2}\right)^{\mu+1 / 2} \Gamma\left(\mu+\frac{1}{2}\right)} \sum_{n=0}^{N} \frac{(-1)^{n} \Gamma\left(\mu+\frac{1}{2}+n\right)}{n !} B\left(\alpha+n, \gamma-\alpha-\mu-\frac{1}{2}-n\right) \delta^{n}+R_{N},
\end{aligned}
$$


which may be written in the form

$$
\begin{aligned}
& R_{\mu}(k, \alpha, \gamma) \\
& \quad=\frac{\Gamma\left(\frac{1}{2}-\mu\right)}{\left(1+k^{2}\right)^{\mu+1 / 2} \Gamma\left(\gamma-\mu-\frac{1}{2}\right)} \sum_{n=0}^{N} \frac{\Gamma(n+\alpha) \Gamma(\gamma-\mu-1 / 2-n)}{n ! \Gamma(1 / 2-\mu-n)} \delta^{n}+R_{N},
\end{aligned}
$$

because

$$
\frac{\Gamma(1 / 2-\mu)}{\Gamma(1 / 2-\mu-n)}=(-1)^{n} \frac{\Gamma(\mu+1 / 2+n)}{\Gamma(\mu+1 / 2)}
$$

for $\operatorname{Re}\left(\gamma-\alpha-\mu-\frac{1}{2}\right)>n, n=0,1, \ldots, N$.

\section{Multiple-term approximations}

3.1. Formulation valid for the parameter $\operatorname{subspace} \operatorname{Re}\left(\mu+\frac{1}{2}+\alpha-\gamma\right)>\mathbf{N}, \mathbf{N} \geq 1$. Assume that there exist complex values $C_{1}, C_{2}, \ldots, C_{s}, z_{1}, z_{2}, \ldots, z_{s}$ such that

$$
\sum_{j=1}^{s} C_{j}(1+x)^{-z_{j}} \approx\left(1-\frac{x}{\kappa}\right)^{\alpha-1},
$$

where $s=1$ for a single-term approximation, $s=2$ for a double-term approximation, and so on. By performing binomial expansions, (7) gives

$$
\sum_{n=0}^{\infty} \sum_{j=1}^{s} C_{j}\left(\begin{array}{c}
-z_{j} \\
n
\end{array}\right) x^{n}=\sum_{n=0}^{\infty}\left(\begin{array}{c}
\alpha-1 \\
n
\end{array}\right)(-1)^{n} \frac{1}{\kappa^{n}} x^{n}
$$

or

$$
\sum_{j=1}^{s} C_{j}\left(z_{j}\right)_{n}=(-1)^{n} \frac{(1-\alpha)_{n}}{\kappa^{n}}, \quad n=0,1,2, \ldots, 2 s-1
$$

where

$$
(a)_{n}=\frac{\Gamma(a+n)}{\Gamma(a)}=a(a+1)(a+1)(a+2) \ldots(a+n-1) \text { and }\left(\begin{array}{c}
-a \\
n
\end{array}\right)=(-1)^{n} \frac{(a)_{n}}{n !} .
$$

3.2. Formulation valid for the parameter subspace $\operatorname{Re}\left(\gamma-\alpha-\mu-\frac{1}{2}\right)>\mathbf{N}, \mathbf{N} \geq$ 1. Assume that there exist complex values $P_{1}, P_{2}, \ldots, P_{s}, x_{1}, x_{2}, \ldots, x_{s}$ such that

$$
\sum_{j=1}^{s} P_{j}(1+\tau)^{-x_{j}} \approx(1+\delta \tau)^{-\mu-1 / 2},
$$

where $s$ determines the number of terms in the approximation. Equation (9) results in

$$
\sum_{j=1}^{s} P_{j}\left(x_{j}\right)_{n}=\left(\mu+\frac{1}{2}\right)_{n} \delta^{n}, \quad n=0,1, \ldots, 2 s-1 .
$$




\section{Single- and double-term approximations}

\subsection{Single-term approximation}

Solution valid for $\operatorname{Re}\left(\mu+\frac{1}{2}+\alpha-\gamma\right) \geq 1$. Set $s=1$ in (8), and $n=0,1$. This results in $C_{1}=1, z_{1}=(\alpha-1) / \kappa$, and (7) is thus

$$
\left(1-\frac{x}{\kappa}\right)^{\alpha-1} \approx(1+x)^{-(\alpha-1) / \kappa} \text {. }
$$

This approximation coincides with that given by Kalla et al. [9]. Upon substitution of this approximation in (3) and performing the integration, the single-term approximation is given by

$$
R_{\mu}(k, \alpha, \gamma) \approx \frac{B\left(\gamma-\alpha, \mu+1 / 2+\alpha-\gamma+\frac{\alpha-1}{\kappa}\right)}{\left(1-k^{2}\right)^{\mu+1 / 2} \kappa^{\gamma-\alpha}}
$$

which has been given by Kalla et al. [9].

Solution valid for $\operatorname{Re}\left(\gamma-\alpha-\mu-\frac{1}{2}\right) \geq 1$. Set $s=1$ in (9), and $n=0,1$. This results in $P_{1}=1, x_{1}=\left(\mu+\frac{1}{2}\right) \delta$. Thus

$$
(1+\delta \tau)^{-\mu-1 / 2} \approx(1+\tau)^{-(\mu+1 / 2) \delta}
$$

which is the same approximation as has been given by the author in [3].

Upon substitution of (13) in (5) and performing the integration, the single-term approximation is given by

$$
R_{\mu}(k, \alpha, \gamma) \approx \frac{B\left(\alpha, \gamma-\alpha-\zeta\left(\mu+\frac{1}{2}\right)\right)}{\left(1+k^{2}\right)^{\mu+1 / 2}}
$$

where $\zeta=2 k^{2} /\left(1+k^{2}\right)$, which is identical to that given by the author in [3].

\subsection{Double-term approximations}

Solution valid for $\operatorname{Re}\left(\mu+\frac{1}{2}+\alpha-\gamma\right)>\mathbf{N}, \mathbf{N} \geq 1$. Set $s=2$ in (8) so that

$$
\sum_{j=1}^{2} C_{j}\left(z_{j}\right)_{n}=(-1)^{n} \frac{(1-\alpha)_{n}}{\kappa_{n}}, \quad n=0,1,2,3 .
$$

This gives a system of four non-linear equations, which can be written in the form

$$
C_{1} z_{1}^{n}+C_{2} z_{2}^{n}=P_{n}, \quad n=0,1,2,3,
$$


where

$$
\begin{aligned}
& p_{0}=1 \\
& p_{1}=\frac{(\alpha-1)}{\kappa} \\
& p_{2}=\frac{(\alpha-1)}{\kappa}\left(\frac{(\alpha-2)}{\kappa}-1\right) \\
& p_{3}=\frac{(\alpha-1)}{\kappa}\left(\frac{(\alpha-2)(\alpha-3)}{\kappa^{2}}-\frac{3(\alpha-2)}{\kappa}+1\right) .
\end{aligned}
$$

Solution with real $\alpha>1$. This solution is obtained by solving the first three equations of (16) for $C_{1}=C_{2}=\frac{1}{2}$ which assumes $z_{1}$ and $z_{2}$ are complex conjugates. Setting $z_{1}=r e^{i \theta}, z_{2}=r e^{-i \theta}$ and solving these equations results in

$$
\begin{aligned}
& r=\frac{\sqrt{(\alpha-1)(\alpha+\kappa)}}{\kappa}, \\
& \theta=\cos ^{-1}\left(\sqrt{\frac{\alpha-1}{\alpha+\kappa}}\right) .
\end{aligned}
$$

Thus

$$
\begin{aligned}
& z_{1}=\frac{(\alpha-1)}{\kappa}+\frac{i \sqrt{(\alpha-1)(1+\kappa)}}{\kappa} \\
& z_{2}=\frac{(\alpha-1)}{\kappa}-\frac{i \sqrt{(\alpha-1)(1+\kappa)}}{\kappa} .
\end{aligned}
$$

Upon substitution of $s=2, C_{1}=C_{2}=\frac{1}{2}$ and (18) in (7) and performing the integration, the double-term approximation for real $\alpha>1$ is given by

$$
R_{\mu}(k, \alpha, \gamma) \approx \frac{B\left(\gamma-\alpha, \mu+1 / 2+\alpha-\gamma+z_{1}\right)+B\left(\gamma-\alpha, \mu+1 / 2+\alpha-\gamma+z_{2}\right)}{2\left(1-k^{2}\right)^{\mu+1 / 2} \kappa^{\gamma-\alpha}}
$$

Solution with complex $\alpha$. The first three equations of (16) are solved for $C_{1}=$ $C_{2}=\frac{1}{2}$ and arbitrary $\alpha, \alpha=\alpha_{x}+i \alpha_{y}$, where $\alpha_{x}$ and $\alpha_{y}$ are the real and imaginary components.

Setting

$$
r_{\alpha}=\sqrt{\left(\alpha_{x}-1\right)^{2}+\alpha_{y}^{2}}, \quad \theta_{\alpha}=\tan ^{-1}\left(\frac{\alpha_{y}}{\alpha_{x}-1}\right)
$$

results in

$$
\begin{aligned}
& z_{1}^{*}=\frac{1}{\kappa}\left\{\alpha_{x}-1-\sqrt{(1+\kappa) r_{\alpha}} \sin \left(\frac{\theta_{\alpha}}{2}\right)\right\}+\frac{i}{\kappa}\left\{\alpha_{y}+\sqrt{(1+\kappa) r_{\alpha}} \cos \left(\frac{\theta_{\alpha}}{2}\right)\right\}, \\
& z_{2}^{*}=\frac{1}{\kappa}\left\{\alpha_{x}-1+\sqrt{(1+\kappa) r_{\alpha}} \sin \left(\frac{\theta_{\alpha}}{2}\right)\right\}+\frac{i}{\kappa}\left\{\alpha_{y}-\sqrt{(1+\kappa) r_{\alpha}} \cos \left(\frac{\theta_{\alpha}}{2}\right)\right\} .
\end{aligned}
$$


By substitution of these values in (7) and performing the integration, the double-term approximation is given by

$$
R_{\mu}(k, \alpha, \gamma) \approx \frac{B\left(\gamma-\alpha, \mu+1 / 2+\alpha-\gamma+z_{1}^{*}\right)+B\left(\gamma-\alpha, \mu+1 / 2+\alpha-\gamma+z_{2}^{*}\right)}{2\left(1-k^{2}\right)^{\mu+1 / 2} \kappa^{\gamma-\alpha}} .
$$

Note that (19) is a special case of (21) when $\alpha$ is real, $\alpha>1$.

An efficient double-term approximation: $\operatorname{Re}\left(\mu+\frac{1}{2}+\alpha-\gamma\right)>\mathbf{N}, \mathbf{N} \geq 1$. The solution of the four equations (16) will be performed as

$$
\begin{gathered}
{\left[\begin{array}{ll}
1 & 1 \\
z_{1} & z_{2}
\end{array}\right]\left[\begin{array}{l}
C_{1} \\
C_{2}
\end{array}\right]=\left[\begin{array}{l}
p_{0} \\
p_{1}
\end{array}\right],} \\
{\left[\begin{array}{ll}
z_{1}^{2} & z_{2}^{2} \\
z_{1}^{3} & z_{2}^{3}
\end{array}\right]\left[\begin{array}{l}
C_{1} \\
C_{2}
\end{array}\right]=\left[\begin{array}{l}
p_{2} \\
p_{3}
\end{array}\right] .}
\end{gathered}
$$

Solving (22) results in

$$
\begin{aligned}
& C_{1}=\frac{z_{2}-p_{1}}{z_{2}-z_{1}}, \\
& C_{2}=\frac{p_{1}-z_{1}}{z_{2}-z_{1}} .
\end{aligned}
$$

Substitution of (24) in (23) leads to

$$
\begin{aligned}
p_{1}\left(z_{1}+z_{2}\right)-z_{1} z_{2} & =p_{2}, \\
p_{1}\left(z_{1}+z_{2}\right)^{2}-z_{1} z_{2}\left[p_{1}+\left(z_{1}+z_{2}\right)\right] & =p_{3} .
\end{aligned}
$$

Setting

$$
\begin{aligned}
z_{1}+z_{2} & =2 p_{s}, \\
z_{1} z_{2} & =p_{p},
\end{aligned}
$$

will resolve the non-linearity in (25) and simplifications result in

$$
\begin{aligned}
& p_{s}=\frac{p_{3}-p_{1} p_{2}}{2\left(p_{2}-p_{1}^{2}\right)}, \\
& p_{p}=\frac{p_{1} p_{3}-p_{2}^{2}}{p_{2}-p_{1}^{2}} .
\end{aligned}
$$

Substitution for $p_{1}, p_{2}$ and $p_{3}$ in (27) results in

$$
\begin{aligned}
& p_{s}=\frac{\alpha-2}{\kappa}-\frac{1}{2}, \\
& p_{p}=\frac{(\alpha-1)(\alpha-2)}{\kappa^{2}} .
\end{aligned}
$$


Finally $z_{1}$ and $z_{2}$ are given by

$$
\begin{aligned}
& z_{1}=p_{s}+\sqrt{p_{s}^{2}+p_{p}}, \\
& z_{2}=p_{s}-\sqrt{p_{s}^{2}+p_{p}} .
\end{aligned}
$$

Substitution of (28) in (29) results in

$$
\begin{aligned}
& z_{1}=\frac{\alpha-2}{\kappa}-\frac{1}{2}+\frac{1}{2} \sqrt{1-\frac{4(\alpha-2)}{\kappa}\left(1+\frac{1}{\kappa}\right)}, \\
& z_{2}=\frac{\alpha-2}{\kappa}-\frac{1}{2}-\frac{1}{2} \sqrt{1-\frac{4(\alpha-2)}{\kappa}\left(1+\frac{1}{\kappa}\right)} .
\end{aligned}
$$

Substitution of (30) and (17) in (24) results in

$$
\begin{aligned}
& C_{1}=\frac{1}{2}+\frac{1 / 2+1 / \kappa}{\sqrt{1-\frac{4(\alpha-2)}{\kappa}\left(1+\frac{1}{\kappa}\right)}}, \\
& C_{2}=\frac{1}{2}-\frac{1 / 2+1 / \kappa}{\sqrt{1-\frac{4(\alpha-2)}{\kappa}\left(1+\frac{1}{\kappa}\right)}} .
\end{aligned}
$$

The double-term approximation in the parameter subspace $\operatorname{Re}\left(\mu+\frac{1}{2}+\alpha-\gamma\right)>$ $N, N \geq 1$ is given by

$$
R_{\mu}(k, \alpha, \gamma) \approx \frac{C_{1} B\left(\gamma-\alpha, \mu+1 / 2+\alpha-\gamma+z_{1}\right)+C_{2} B\left(\gamma-\alpha, \mu+1 / 2+\alpha-\gamma+z_{2}\right)}{\left(1-k^{2}\right)^{\mu+1 / 2} \kappa^{\gamma-\alpha}} .
$$

Note that as $\kappa \rightarrow \infty, z_{1} \rightarrow 0, z_{2} \rightarrow-1, C_{1} \rightarrow 1, C_{2} \rightarrow 0$ and $(1-x / \kappa)^{\alpha-1} \rightarrow 1$, a requirement for convergence.

Solution with $\operatorname{Re}\left(\gamma-\alpha-\mu-\frac{1}{2}\right)>\mathbf{N}, \mathbf{N} \geq 1$. Set $s=2$ in (10) so that

$$
\sum_{j=1}^{2} P_{j}\left(x_{j}\right)_{n}=\left(\mu+\frac{1}{2}\right)_{n} \delta^{n} \quad n=0,1,2,3
$$

which gives a system of four non-linear equations that can be written as

$$
P_{1} x_{1}^{n}+P_{2} x_{2}^{n}=e_{n} \quad n=0,1,2,3,
$$


where

$$
\begin{aligned}
& e_{0}=1 \\
& e_{1}=\left(\mu+\frac{1}{2}\right) \delta \\
& e_{2}=\left(\mu+\frac{1}{2}\right) \delta\left(\left(\mu+\frac{3}{2}\right) \delta-1\right) \\
& e_{3}=\left(\mu+\frac{1}{2}\right) \delta\left(\left(\mu+\frac{3}{2}\right)\left(\mu+\frac{5}{2}\right) \delta^{2}-3\left(\mu+\frac{3}{2}\right) \delta+1\right) .
\end{aligned}
$$

Solution with real $\mu$. This solution is obtained by solving the first three equations of (34) for $P_{1}=P_{2}=\frac{1}{2}$, where in this case $x_{1}$ and $x_{2}$ are complex conjugates. Solving these equations results in

$$
\begin{aligned}
& x_{1}=\left(\mu+\frac{1}{2}\right) \delta+i \sqrt{\left(\mu+\frac{1}{2}\right) \zeta \delta}, \\
& x_{2}=\left(\mu+\frac{1}{2}\right) \delta-i \sqrt{\left(\mu+\frac{1}{2}\right) \zeta \delta} .
\end{aligned}
$$

Upon substitution of $s=2, P_{1}=P_{2}=\frac{1}{2}$ and (35) in (9) and performing the integration, the double-term approximation for real $\mu$ is given by

$$
R_{\mu}(k, \alpha, \gamma) \approx \frac{B\left(\alpha, \gamma-\alpha-\mu-1 / 2+x_{1}\right)+B\left(\alpha, \gamma-\alpha-\mu-1 / 2+x_{2}\right)}{2\left(1+k^{2}\right)^{\mu+1 / 2}} .
$$

Solution with complex $\mu$. The first three equations (34) are solved for $P_{1}=$ $P_{2}=\frac{1}{2}, \mu=\mu_{x}+i \mu_{y}$, where $\mu_{x}$ and $\mu_{y}$ are the real and imaginary components of $\mu$. Setting

$$
r_{\mu}=\sqrt{\left(\mu_{x}+1 / 2\right)^{2}+\mu_{y}^{2}}, \quad \theta_{\mu}=\tan ^{-1}\left(\frac{\mu_{y}}{\mu_{x}+1 / 2}\right)
$$

results in

$$
\begin{aligned}
& x_{1}^{*}=\left(\mu_{x}+\frac{1}{2}\right) \delta-\sqrt{r_{\mu} \zeta \delta} \sin \left(\frac{\theta_{\mu}}{2}\right)+i\left\{\mu_{y} \delta+\sqrt{r_{\mu} \zeta \delta} \cos \left(\frac{\theta_{\mu}}{2}\right)\right\}, \\
& x_{2}^{*}=\left(\mu_{x}+\frac{1}{2}\right) \delta+\sqrt{r_{\mu} \zeta \delta} \sin \left(\frac{\theta_{\mu}}{2}\right)+i\left\{\mu_{y} \delta-\sqrt{r_{\mu} \zeta \delta} \cos \left(\frac{\theta_{\mu}}{2}\right)\right\} .
\end{aligned}
$$

On substitution of these values in (9) and performing the integration, the double-term approximation is given by

$$
R_{\mu}(k, \alpha, \gamma) \approx \frac{B\left(\alpha, \gamma-\alpha-\mu-1 / 2+x_{1}^{*}\right)+B\left(\alpha, \gamma-\alpha-\mu-1 / 2+x_{2}^{*}\right)}{2\left(1+k^{2}\right)^{\mu+1 / 2}}
$$

Note that (36) is a special case of (38) when $\mu$ is real. 
An efficient double-term approximation $\operatorname{Re}(\gamma-\alpha-\mu-1 / 2)>N, N \geq 1$. The solution of the four equations (34) will be performed as

$$
\begin{gathered}
{\left[\begin{array}{cc}
1 & 1 \\
x_{1} & x_{2}
\end{array}\right]\left[\begin{array}{l}
P_{1} \\
P_{2}
\end{array}\right]=\left[\begin{array}{l}
e_{0} \\
e_{1}
\end{array}\right],} \\
{\left[\begin{array}{ll}
x_{1}^{2} & x_{2}^{2} \\
x_{1}^{3} & x_{2}^{3}
\end{array}\right]\left[\begin{array}{l}
P_{1} \\
P_{2}
\end{array}\right]=\left[\begin{array}{l}
e_{2} \\
e_{3}
\end{array}\right] .}
\end{gathered}
$$

The solution is obtained using a similar approach to the previous case, which results in

$$
\begin{gathered}
x_{1}=\left(\mu+\frac{3}{2}\right) \delta-\frac{1}{2}+\frac{1}{2} \sqrt{1-4\left(\mu+\frac{3}{2}\right) \zeta \delta} \\
x_{2}=\left(\mu+\frac{3}{2}\right) \delta-\frac{1}{2}-\frac{1}{2} \sqrt{1-4\left(\mu+\frac{3}{2}\right) \zeta \delta} \\
P_{1}=\frac{1}{2}+\frac{\left(\frac{1}{2}-\delta\right)}{\sqrt{1-4\left(\mu+\frac{3}{2}\right) \zeta \delta}} \\
P_{2}=\frac{1}{2}-\frac{\left(\frac{1}{2}-\delta\right)}{\sqrt{1-4\left(\mu+\frac{3}{2}\right) \zeta \delta}} .
\end{gathered}
$$

The double-term approximation is given by

$$
R_{\mu}(k, \alpha, \gamma) \approx \frac{P_{1} B\left(\alpha, \gamma-\alpha-\mu-1 / 2+x_{1}\right)+i P_{2} B\left(\alpha, \gamma-\alpha-\mu-1 / 2+x_{2}\right)}{2\left(1+k^{2}\right)^{\mu+1 / 2}} .
$$

Note that as $\delta \rightarrow 0, x_{1} \rightarrow 0, x_{2} \rightarrow-1, P_{1} \rightarrow 1, P_{2} \rightarrow 0$ and $(1+\delta \tau)^{\mu+1 / 2} \rightarrow 1$ and convergence is guaranteed.

\section{Results and conclusions}

In this section the implementation issues, error estimates and results are considered, and concluding remarks made.

The proposed algorithm has been implemented using a high-level programming language with extended memory (better than double precision) for highly accurate results. Furthermore this implementation allows the user to enter complex data values 
using batch/interactive (user friendly) modes of operation. Thus different computer runs have been made for some real as well as for some complex parameter values $\mu, k \rightarrow 1, \alpha$ and $\gamma$. The approximation formulas for $R_{\mu}(k, \alpha, \gamma)$ developed by the author are given in terms of the well known beta function. Since the arguments of the beta function should have positive real part (from its integral definition), different approximation formulas with different error measures ranging from o $(h)$ to $o\left(h^{2 s-1}\right)$ ( $s=1$ for single term, $s=2$ for double term, and so on) have been developed to meet this requirement for an arbitrary selection of parameter values. Implementation has been performed for single and double term approximations. Our formulation can be extended to multiple term approximations by selecting $s=3,4, \ldots, n$ in (8) and (10).

Error estimates for the different formulas implemented for [8, (24)], single-, and double-term approximations are as follows (note that $\mathrm{O}$ stands for big order and $\mathrm{o}$ stands for small order).

Equation $[8,(24)]$ is a first-order approximation with error of the order of $O(1 / \kappa)$. For single-term approximations:

(i) the error in approximating (1) using (12) is o( $1 / \kappa)$.

(ii) the error in approximating (1) using (14) is $o(\delta)$.

For double-term approximations:

(i) the error in approximating (1) using (19), and (21) is o $\left(1 / \kappa^{2}\right)$.

(ii) the error in approximating (1) using (36), and (39) is $o\left(\delta^{2}\right)$.

(iii) the error in approximating (1) using (32) is $o\left(1 / \kappa^{3}\right)$.

(iv) the error in approximating (1) using (43) is $o\left(\delta^{3}\right)$.

Comparisons of the numerical values of $R_{\mu}(k, \alpha, \gamma)$ near $k^{2}=1$ computed using different formulas are shown in Tables 1 and 2 for some real parameter values and in Tables 3 and 4 for some complex parameter values. These results shows the efficiency of the derived algorithms for an arbitrary valid selection of parameter values in the range $\left|\operatorname{Re}\left(\mu+\frac{1}{2}+\alpha-\gamma\right)\right|>1$. In this range the results agree fully with the error estimates described above, in which (32) and (44) are the most accurate ones. However, in the parameter subspace where $\left|\operatorname{Re}\left(\mu+\frac{1}{2}+\alpha-\gamma\right)\right|$ is near unity (where neither approximation is valid) the results are still acceptable for the two-term approximations (19), (21), (36), and (38).

\section{Acknowledgement}

The author thanks the reviewer for his valuable comments and Professor S. L. Kalla for his encouragement. 
TABLE 1. Comparison of the numerical values of $R_{\mu}(k, \alpha, \gamma)$ near $k^{2}=1$ for some real parameter values in the range $\gamma-\alpha-\mu>0.0$ computed using [8,(21)], [8,(24)], (14), (39), and (44) respectively.

(1) $\alpha=1.100, \gamma=4.200, \mu=0.500, \gamma-\alpha-\mu-1 / 2=2.1$

\begin{tabular}{lccccc}
$k$ & {$[8,(21)]$} & {$[8,(24)]$} & Eqn (14) & Eqn (39) & Eqn (44) \\
\hline 0.9 & $2.093934 \mathrm{E}-01$ & $1.838617 \mathrm{E}-01$ & $2.152868 \mathrm{E}-01$ & $2.107449 \mathrm{E}-01$ & $2.082308 \mathrm{E}-01$ \\
0.99 & $2.054540 \mathrm{E}-01$ & $2.091932 \mathrm{E}-01$ & $2.063600 \mathrm{E}-01$ & $2.058484 \mathrm{E}-01$ & $2.053967 \mathrm{E}-01$ \\
0.999 & $2.053540 \mathrm{E}-01$ & $2.057701 \mathrm{E}-01$ & $2.054612 \mathrm{E}-01$ & $2.054095 \mathrm{E}-01$ & $2.053617 \mathrm{E}-01$
\end{tabular}

(2) $\alpha=1.100, \gamma=4.200, \mu=0.800, \gamma-\alpha-\mu-1 / 2=1.8$

\begin{tabular}{lccccc}
$k$ & {$[8,(21)]$} & {$[8,(24)]$} & Eqn (14) & Eqn (39) & Eqn (44) \\
\hline 0.9 & $1.979907 \mathrm{E}-01$ & $3.470349 \mathrm{E}-01$ & $2.072572 \mathrm{E}-01$ & $2.000121 \mathrm{E}-01$ & $1.951857 \mathrm{E}-01$ \\
0.99 & $1.963101 \mathrm{E}-01$ & $2.029821 \mathrm{E}-01$ & $1.979592 \mathrm{E}-01$ & $1.971006 \mathrm{E}-01$ & $1.960783 \mathrm{E}-01$ \\
0.999 & $1.968272 \mathrm{E}-01$ & $1.974540 \mathrm{E}-01$ & $1.970314 \mathrm{E}-01$ & $1.969441 \mathrm{E}-01$ & $1.968335 \mathrm{E}-01$
\end{tabular}

(3) $\alpha=4.100, \gamma=6.600, \mu=0.500, \gamma-\alpha-\mu-1 / 2=1.5$

\begin{tabular}{lccccc}
$k$ & {$[8,(21)]$} & {$[8,(24)]$} & Eqn (14) & Eqn (39) & Eqn (44) \\
\hline 0.9 & $3.782468 \mathrm{E}-02$ & $1.384385 \mathrm{E}-01$ & $4.602029 \mathrm{E}-02$ & $3.992645 \mathrm{E}-02$ & $3.193469 \mathrm{E}-02$ \\
0.99 & $4.670842 \mathrm{E}-02$ & $5.167806 \mathrm{E}-02$ & $4.875022 \mathrm{E}-02$ & $4.796716 \mathrm{E}-02$ & $4.581456 \mathrm{E}-02$ \\
0.999 & $4.873123 \mathrm{E}-02$ & $4.919103 \mathrm{E}-02$ & $4.901376 \mathrm{E}-02$ & $4.893351 \mathrm{E}-02$ & $4.869304 \mathrm{E}-02$
\end{tabular}

(4) $\alpha=1.100, \gamma=2.800, \mu=0.500, \gamma-\alpha-\mu-1 / 2=0.7$

\begin{tabular}{lccccc}
$k$ & {$[8,(21)]$} & {$[8,(24)]$} & Eqn (14) & Eqn (39) & Eqn (44) \\
\hline 0.9 & $5.293588 \mathrm{E}-01$ & $2.837057 \mathrm{E}-01$ & $6.321023 \mathrm{E}-01$ & $5.461978 \mathrm{E}-01$ & $2.523377 \mathrm{E}-02$ \\
0.99 & $6.148831 \mathrm{E}-01$ & $5.966988 \mathrm{E}-01$ & $6.596220 \mathrm{E}-01$ & $6.459745 \mathrm{E}-01$ & $6.988331 \mathrm{E}-01$ \\
0.999 & $6.504273 \mathrm{E}-01$ & $6.488000 \mathrm{E}-01$ & $6.626075 \mathrm{E}-01$ & $6.611700 \mathrm{E}-01$ & $6.660787 \mathrm{E}-01$
\end{tabular}

(5) $\alpha=1.100, \gamma=3.000, \mu=0.800, \gamma-\alpha-\mu-1 / 2=0.6$

\begin{tabular}{lccccr}
$k$ & {$[8,(21)]$} & {$[8,(24)]$} & Eqn (14) & Eqn (39) & \multicolumn{1}{c}{ Eqn (44) } \\
\hline 0.8 & $4.295741 \mathrm{E}-01$ & $-2.115114 \mathrm{E}-01$ & $5.437709 \mathrm{E}-01$ & $4.143580 \mathrm{E}-01$ & $2.871081 \mathrm{E}-01$ \\
0.85 & $4.391267 \mathrm{E}-01$ & $5.399295 \mathrm{E}-02$ & $5.609462 \mathrm{E}-01$ & $4.337491 \mathrm{E}-01$ & $1.841555 \mathrm{E}-01$ \\
0.9 & $4.563970 \mathrm{E}-01$ & $2.479054 \mathrm{E}-01$ & $5.811327 \mathrm{E}-01$ & $4.669419 \mathrm{E}-01$ & $-3.444037 \mathrm{E}-01$
\end{tabular}

(6) $\alpha=1.100, \gamma=2.600, \mu=0.500, \gamma-\alpha-\mu-1 / 2=0.5$

\begin{tabular}{lccccr}
$k$ & {$[8,(21)]$} & {$[8,(24)]$} & Eqn (14) & Eqn (39) & \multicolumn{1}{c}{ Eqn (44) } \\
\hline 0.8 & $6.052904 \mathrm{E}-01$ & $1.727362 \mathrm{E}-01$ & $7.853774 \mathrm{E}-01$ & $5.769254 \mathrm{E}-01$ & $2.798452 \mathrm{E}-01$ \\
0.85 & $6.233944 \mathrm{E}-01$ & $3.532578 \mathrm{E}-01$ & $8.174955 \mathrm{E}-01$ & $6.119775 \mathrm{E}-01$ & $-1.357250 \mathrm{E}-01$ \\
0.9 & $6.525245 \mathrm{E}-01$ & $5.007962 \mathrm{E}-01$ & $8.540038 \mathrm{E}-01$ & $6.688503 \mathrm{E}-01$ & $5.618882 \mathrm{E}+00$ \\
\hline
\end{tabular}


TABLE 2. Comparison of the numerical values of $R_{\mu}(k, \alpha, \gamma)$ near $k^{2}=1$ for some real parameter values in the range $\alpha+\mu-\gamma>0.0$ computed using [8,(21)], [8,(24)], (12), (19), and (32) respectively.

\begin{tabular}{lccccc}
$k$ & {$[8,(21)]$} & {$[8,(24)]$} & Eqn $(12)$ & Eqn (19) & Eqn (32) \\
\hline$(1) \alpha=0.900, \gamma=1.000, \mu=0.850, \alpha+\mu+1 / 2-\gamma=2.1$ \\
0.99 & $1.210422 \mathrm{E}+03$ & $1.209931 \mathrm{E}+03$ & $1.210498 \mathrm{E}+03$ & $1.210581 \mathrm{E}+03$ & $1.210886 \mathrm{E}+03$ \\
0.999 & $2.136422 \mathrm{E}+04$ & $2.136337 \mathrm{E}+04$ & $2.136403 \mathrm{E}+04$ & $2.136417 \mathrm{E}+04$ & $2.136465 \mathrm{E}+04$ \\
0.9999 & $3.796268 \mathrm{E}+05$ & $3.796253 \mathrm{E}+05$ & $3.796262 \mathrm{E}+05$ & $3.796264 \mathrm{E}+05$ & $3.796272 \mathrm{E}+05$ \\
\hline$(2) \alpha=5.900, \gamma=6.200, \mu=2.000, \alpha+\mu+1 / 2-\gamma=2.20$ \\
0.99 & $1.106853 \mathrm{E}+04$ & $1.118958 \mathrm{E}+04$ & $1.111249 \mathrm{E}+04$ & $1.108410 \mathrm{E}+04$ & $1.106495 \mathrm{E}+04$ \\
0.999 & $1.745831 \mathrm{E}+06$ & $1.747946 \mathrm{E}+06$ & $1.746595 \mathrm{E}+06$ & $1.746135 \mathrm{E}+06$ & $1.745819 \mathrm{E}+06$ \\
0.9999 & $2.765751 \mathrm{E}+08$ & $2.766086 \mathrm{E}+08$ & $2.765871 \mathrm{E}+08$ & $2.765798 \mathrm{E}+08$ & $2.765747 \mathrm{E}+08$ \\
\hline$(3) \alpha=1.100, \gamma=2.800, \mu=3.500, \alpha+\mu+1 / 2-\gamma=2.30$ \\
0.9 & $3.444503 \mathrm{E}+00$ & $3.506083 \mathrm{E}+00$ & $3.520160 \mathrm{E}+00$ & $3.504459 \mathrm{E}+00$ & $3.483416 \mathrm{E}+00$ \\
0.99 & $4.595303 \mathrm{E}+02$ & $4.601659 \mathrm{E}+02$ & $4.598788 \mathrm{E}+02$ & $4.597165 \mathrm{E}+02$ & $4.595665 \mathrm{E}+02$ \\
0.999 & $8.810573 \mathrm{E}+04$ & $8.811750 \mathrm{E}+04$ & $8.811173 \mathrm{E}+04$ & $8.810868 \mathrm{E}+04$ & $8.810596 \mathrm{E}+04$ \\
\hline$(4) \alpha=1.800, \gamma=3.800, \mu=4.900, \alpha+\mu+1 / 2-\gamma=2.40$ \\
0.9 & $6.640246 \mathrm{E}+00$ & $7.225418 \mathrm{E}+00$ & $6.875321 \mathrm{E}+00$ & $6.736296 \mathrm{E}+00$ & $6.641078 \mathrm{E}+00$ \\
0.99 & $1.050541 \mathrm{E}+04$ & $1.057719 \mathrm{E}+04$ & $1.053254 \mathrm{E}+04$ & $1.051492 \mathrm{E}+04$ & $1.050549 \mathrm{E}+04$ \\
0.999 & $2.521706 \mathrm{E}+07$ & $2.523399 \mathrm{E}+07$ & $2.522345 \mathrm{E}+07$ & $2.521931 \mathrm{E}+07$ & $2.521714 \mathrm{E}+07$ \\
\hline$(5) \alpha=0.700, \gamma=1.000, \mu=3.00, \alpha+\mu+1 / 2-\gamma$ &
\end{tabular}

(5) $\alpha=0.700, \gamma=1.000, \mu=3.000, \alpha+\mu+1 / 2-\gamma=3.20$

$\begin{array}{llllll}0.9 & 3.856661 \mathrm{E}+02 & 3.835179 \mathrm{E}+02 & 3.851123 \mathrm{E}+02 & 3.854767 \mathrm{E}+02 & 3.856644 \mathrm{E}+02\end{array}$

$\begin{array}{lllllll}0.99 & 4.954628 \mathrm{E}+05 & 4.952559 \mathrm{E}+05 & 4.954134 \mathrm{E}+05 & 4.954492 \mathrm{E}+05 & 4.954628 \mathrm{E}+05\end{array}$

$\begin{array}{lllllll}0.999 & 7.695165 \mathrm{E}+08 & 7.694855 \mathrm{E}+08 & 7.695096 \mathrm{E}+08 & 7.695150 \mathrm{E}+08 & 7.695170 \mathrm{E}+08\end{array}$

(6) $\alpha=0.450, \gamma=2.300, \mu=5.500, \alpha+\mu+1 / 2-\gamma=4.15$

$\begin{array}{lllllll}0.9 & 2.411059 \mathrm{E}+01 & 2.299221 \mathrm{E}+01 & 2.371229 \mathrm{E}+01 & 2.394027 \mathrm{E}+01 & 2.406342 \mathrm{E}+01\end{array}$

$\begin{array}{lllllll}0.99 & 1.898106 \mathrm{E}+05 & 1.891848 \mathrm{E}+05 & 1.896169 \mathrm{E}+05 & 1.897551 \mathrm{E}+05 & 1.898105 \mathrm{E}+05\end{array}$

$\begin{array}{lllllll}0.999 & 2.537167 \mathrm{E}+09 & 2.536363 \mathrm{E}+09 & 2.536933 \mathrm{E}+09 & 2.537113 \mathrm{E}+09 & 2.537184 \mathrm{E}+09\end{array}$

(7) $\alpha=0.700, \gamma=1.200, \mu=5.300, \alpha+\mu+1 / 2-\gamma=4.3$

$\begin{array}{lllllll}0.9 & 4.133850 \mathrm{E}+03 & 4.116341 \mathrm{E}+03 & 4.130749 \mathrm{E}+03 & 4.133158 \mathrm{E}+03 & 4.133785 \mathrm{E}+03\end{array}$

$\begin{array}{llllllll}0.99 & 5.844504 \mathrm{E}+08 & 5.842442 \mathrm{E}+08 & 5.844200 \mathrm{E}+08 & 5.844463 \mathrm{E}+08 & 5.844517 \mathrm{E}+08\end{array}$

$\begin{array}{lllllll}0.999 & 1.127955 \mathrm{E}+14 & 1.127918 \mathrm{E}+14 & 1.127952 \mathrm{E}+14 & 1.127957 \mathrm{E}+14 & 1.127958 \mathrm{E}+14\end{array}$

(8) $\alpha=4.000, \gamma=6.500, \mu=8.5000, \alpha+\mu+1 / 2-\gamma=6.50$

$\begin{array}{llllll}0.9 & 1.183240 \mathrm{E}+02 & 1.385660 \mathrm{E}+02 & 1.229674 \mathrm{E}+02 & 1.192964 \mathrm{E}+02 & 1.181464 \mathrm{E}+02\end{array}$

$\begin{array}{lllllll}0.99 & 1.986265 \mathrm{E}+08 & 2.013939 \mathrm{E}+08 & 1.992768 \mathrm{E}+08 & 1.987617 \mathrm{E}+08 & 1.986258 \mathrm{E}+08\end{array}$

$0.999 \quad 5.902838 \mathrm{E}+14 \quad 5.910941 \mathrm{E}+14 \quad 5.904770 \mathrm{E}+14 \quad 5.903264 \mathrm{E}+14 \quad 5.902874 \mathrm{E}+14$

(9) $\alpha=1.200, \gamma=3.400, \mu=10.000, \alpha+\mu+1 / 2-\gamma=8.30$

$\begin{array}{lllllll}0.9 & 3.003545 \mathrm{E}+03 & 3.025522 \mathrm{E}+03 & 3.007982 \mathrm{E}+03 & 3.004467 \mathrm{E}+03 & 3.003632 \mathrm{E}+03\end{array}$

$\begin{array}{llllllll}0.95 & 6.038944 \mathrm{E}+05 & 6.058987 \mathrm{E}+05 & 6.042773 \mathrm{E}+05 & 6.039695 \mathrm{E}+05 & 6.039052 \mathrm{E}+05\end{array}$

$\begin{array}{lllllll}0.999 & 4.991774 \mathrm{E}+19 & 4.992153 \mathrm{E}+19 & 4.991905 \mathrm{E}+19 & 4.991860 \mathrm{E}+19 & 4.991852 \mathrm{E}+19\end{array}$ 
TABLE 3. Comparison of the numerical values of $R_{\mu}(k, \alpha, \gamma)$ near $k^{2}=1$ for some complex parameter values in the range $\alpha+\mu-\gamma>0.0$ computed using [8,(21)], [8,(24)], (12), (21), and (32) respectively.

(1) $\alpha=(0.900,0.700), \gamma=(1.000,0.800), \mu=(0.850,0.600), \operatorname{Re}(\alpha+\mu+1 / 2-\gamma)=1.25$

\begin{tabular}{ccc}
$k$ & {$[8,(21)]$} & {$[8,(24)]$} \\
\hline 0.900 & $(53.233625,-6.280356)$ & $(53.534379,-5.278908)$ \\
0.990 & $(473.282878,747.731133)$ & $(472.109255,748.812009)$ \\
0.999 & $(-8620.248592,13028.77982)$ & $(-8622.831955,13027.68588)$ \\
$k$ & Eqn $(12)$ & Eqn $(21)$ \\
\hline 0.900 & $(53.630786,-6.008611)$ & $(53.532530,-6.336027)$ \\
0.990 & $(472.931028,748.560205)$ & $(473.324386,748.297231)$ \\
0.999 & $(-8622.015485,13028.87515)$ & $(-8621.325519,13029.31969)$ \\
$k$ & Eqn $(32)$ & \\
\hline 0.900 & $(53.038156,-6.203533)$ & \\
0.990 & $(473.195063,747.613208)$ & \\
0.999 & $(-8620.313258,13028.68662)$ &
\end{tabular}

(2) $\alpha=(0.700,0.800), \gamma=(1.000,0.700), \mu=(3.000,1.700), \operatorname{Re}(\alpha+\mu+1 / 2-\gamma)=3.2$

\begin{tabular}{ccc}
$k$ & {$[8,(21)]$} & {$[8,(24)]$} \\
\hline 0.800 & $(-24.000830,37.553806)$ & $(-25.159671,37.763667)$ \\
0.900 & $(-319.160376,-58.900526)$ & $(-320.929155,-62.063334)$ \\
0.990 & $(180265.7725,381195.851)$ & $(180081.096,381562.560)$ \\
$k$ & Eqn $(12)$ & Eqn $(21)$ \\
\hline 0.800 & $(-24.158842,37.801935)$ & $(-23.974962,37.645515)$ \\
0.900 & $(-319.922950,-59.161555)$ & $(-319.370876,-58.810854)$ \\
0.990 & $(180281.422,381282.123)$ & $(180281.297,381214.797)$ \\
$k$ & Eqn $(32)$ & \\
\hline 0.800 & $(-23.987450,37.533648)$ & \\
0.900 & $(-319.144264,-58.874237)$ & $(8,24)]$ \\
0.990 & $(180267.936,381200.619)$ & $(60.173725,62.276061)$ \\
& & $(103325460.9,71869786.4)$ \\
$(3) \alpha=(4.000,1.800), \gamma=(6.500,2.000), \mu$ & $(8.500,5.700), \operatorname{Re}(\alpha+\mu+1 / 2-\gamma)=6.5$ \\
$k$ & {$[8,(21)]$} & $(2.87902 \mathrm{E}+14,2.31463 \mathrm{E}+14)$ \\
\hline 0.900 & $(51.695508,55.877749)$ & Eqn $(21)$ \\
0.990 & $(102034016.4,71234905.9)$ & $(51.983772,55.723464)$ \\
0.999 & $(2.87549 \mathrm{E}+14,2.31198 \mathrm{E}+14)$ & $(102063909.5,71225830.9)$ \\
$k$ & Eqn $(12)$ & $(2.87548 \mathrm{E}+14,2.31251 \mathrm{E}+14)$ \\
\hline 0.900 & $(53.659487,56.020544)$ & \\
0.990 & $(102297179.4,71216634.1)$ & \\
0.999 & $(2.87617 \mathrm{E}+14,2.3125279 \mathrm{E}+14)$ & \\
$k$ & Eqn $(32)$ & \\
\hline 0.900 & $(51.659125,55.923562)$ & \\
0.990 & $(102031838.8,71256335.9)$ & \\
0.999 & $(2.87539 \mathrm{E}+14,2.31259 \mathrm{E}+14)$ & \\
\hline & &
\end{tabular}


TABLE 4. Comparison of the numerical values of $R_{\mu}(k, \alpha, \gamma)$ near $k^{2}=1$ for some complex parameter values in the range $\gamma-\alpha-\mu>0.0$ computed using [8,(21)], [8,(24)], (14), (39), and (44) respectively.

(1) $\alpha=(1.800,2.200), \gamma=(3.800,2.500), \mu=(0.800,0.600), \operatorname{Re}(\gamma-\alpha-\mu-1 / 2)=0.7$

\begin{tabular}{ccc}
$k$ & {$[8,(21)]$} & {$[8,(24)]$} \\
\hline 0.900 & $(0.062210,-0.133610)$ & $(-0.180580,-0.115165)$ \\
0.990 & $(0.145507,-0.109602)$ & $(0.129122,-0.111037)$ \\
0.999 & $(0.167232,-0.082968)$ & $(0.165698,-0.083159)$ \\
$k$ & Eqn (14) & Eqn (39) \\
\hline 0.900 & $(0.137686,-0.105474)$ & $(0.159704,-0.085353)$ \\
0.990 & $(0.164314,-0.075990)$ & $(0.166175,-0.073468)$ \\
0.999 & $(0.166592,-0.072469)$ & \\
$k$ & Eqn $(44)$ & \\
\hline 0.900 & $(0.046495,-0.445326)$ & \\
0.990 & $(0.189366,-0.071709)$ & \\
0.999 & $(0.168855,-0.071864)$ &
\end{tabular}

(2) $\alpha=(3.100,2.300), \gamma=(3.500,4.100), \mu=(1.500,0.900), \operatorname{Re}(\gamma-\alpha-\mu-1 / 2)=1.6$

\begin{tabular}{|c|c|c|}
\hline$k$ & {$[8,(21)]$} & {$[8,(24)]$} \\
\hline 0.900 & $(-0.690440,2.419067)$ & $(0.741511,1.768056)$ \\
\hline 0.990 & $(50.592918,3.185830)$ & $(48.064994,2.901764)$ \\
\hline 0.999 & $(-845.293873,-1641.763390)$ & $(-841.198662,-1633.025388)$ \\
\hline$k$ & Eqn (14) & Eqn (39) \\
\hline 0.900 & $(-0.838484,1.498341)$ & $(-0.927487,1.995796)$ \\
\hline 0.990 & $(47.918409,1.126190)$ & $(49.261647,1.232497)$ \\
\hline 0.999 & $(-849.303877,-1634.613743)$ & $(-851.247429,-1639.355073)$ \\
\hline$k$ & Eqn (44) & \\
\hline 0.900 & $(-1.694687,2.291769)$ & \\
\hline 0.990 & $(49.431664,2.677346)$ & \\
\hline 0.999 & $(-846.762018,-1642.202329)$ & \\
\hline \multicolumn{3}{|c|}{ (3) $\alpha=(1.100,0.600), \gamma=(2.800,1.600), \mu=(3.500,2.700), \operatorname{Re}(\gamma-\alpha-\mu-1 / 2)=2.3$} \\
\hline$k$ & {$[8,(21)]$} & {$[8,(24)]$} \\
\hline 0.900 & $(2.997343,0.547758)$ & $(3.080951,0.737646)$ \\
\hline 0.990 & $(-304.024857,-273.367087)$ & $(-303.606312,-275.681007)$ \\
\hline 0.999 & $(6826.118865,78204.919249)$ & $(6787.219163,78232.622919)$ \\
\hline$k$ & Eqn (14) & Eqn (39) \\
\hline 0.900 & $(3.059542,0.602605)$ & $(3.030089,0.549819)$ \\
\hline 0.990 & $(-304.198135,-274.317901)$ & $(-304.237090,-273.672673)$ \\
\hline 0.999 & $(6812.929814,78221.307795)$ & $(6821.757142,78212.955010)$ \\
\hline$k$ & Eqn (44) & \\
\hline 0.900 & $(2.989206,0.537953)$ & \\
\hline 0.990 & $(-304.022982,-273.379601)$ & \\
\hline 0.999 & $(6822.616646,78206.332409)$ & \\
\hline
\end{tabular}




\section{References}

[1] B. Al-Saqabi, “A generalization of elliptic-type integrals", Hadronic J. 10 (1987) 331-337.

[2] M. J. Berger and J. C. Lamkin, "Sample calculation of Gamma ray penetration into shelters contribution of sky shine and roof contamination", J. Res. NBS 60 (1958) 109-116.

[3] M. El-Gabali, "Single term asymptotic approximations for the generalized elliptic-type integrals", J. Revista de la Academia Canaria da Ciencias 6 (1994), (fasc. 1-1994) 105-118.

[4] L. F. Epstein and J. H. Hubbell, "Evaluation of a generalised elliptic type integral", J. Res. NBS Ser. B 67 (1963) 1-17.

[5] J. H. Hubbell, R. L. Bach and R. J. Herbold, "Radiation field from a circular disc source", J. Res. NBS Ser. C 65 (1961) 249-264.

[6] S. L. Kalla, "Results on generalised elliptic-type integrals", in Mathematical Structures - Computational Mathematics - Mathematical Modelling (ed. Bl. Sendov), second ed., (special vol.), (Bulg. Acad. Sci, 1984) 216-219.

[7] S. L. Kalla and B. Al-Saqabi, "On a generalized elliptic-type integral”, Rev. Bra. Fis. 16 (1986) 145-156.

[8] S. L. Kalla, S. Conde and J. H. Hubbell, "Some results on generalized elliptic-type integrals", Appl. Anal. 22 (1986) 273-287.

[9] S. L. Kalla, C. Leubner and J. H. Hubbell, "Further results on generalized elliptic-type integrals", Appl. Anal. 25 (1987) 269-274. 